\title{
Composite Technical Solutions, Program Products and Complex Integrative Technical Systems Containing Them, as the Underlying Basis for the Modification of the Algorithm for Inventive Problem Solving while Creating Equivalents of Complex Integrative Inventions
}

\author{
Konstantin A. Piliugin, Ph.D. \\ Voronezh, Russian Federation
}

Continuous complication of new developments, in preparation of all stages of their design and classification analytical stages of evaluation, requires patent search and commercialization stages, new managers and corrective analytical tools similar in efficiency, versatility, and significance to the Algorithm for Inventive Problem Solving known in professional innovation groups.

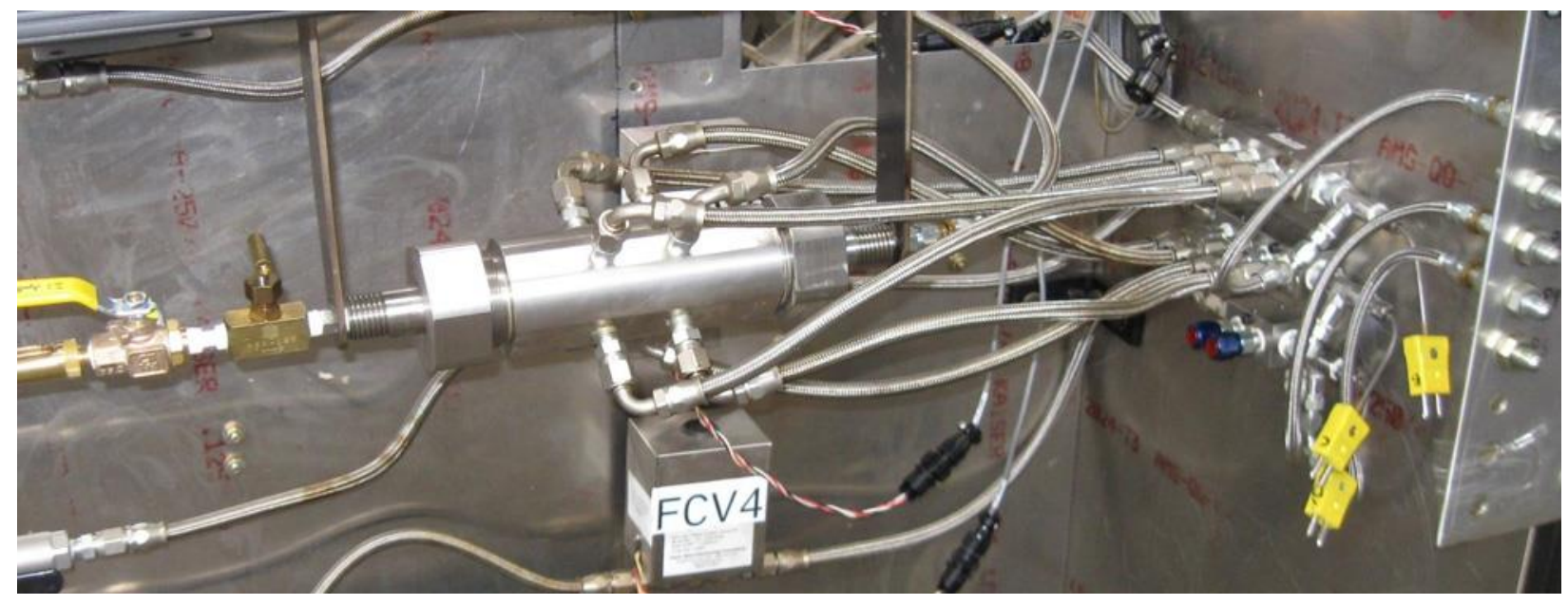

Figure 1. An example of a composite technical solution (developed integrative technical system) designed to optimize the hydrodynamic parameters of the fluid flow in a pipeline, including stabilization of the basic and auxiliary hydrodynamic parameters of the fluid flow in a developed turbulent flow where turbulence levels under standard conditions in the center and on the periphery of the pipe are significantly different. An example is shown on the prototype of the above technical solution, installed on a test bench to verify correctness of the input calculations and assumptions, and subsequent tests and comparative verification of the adopted conceptual solutions. 


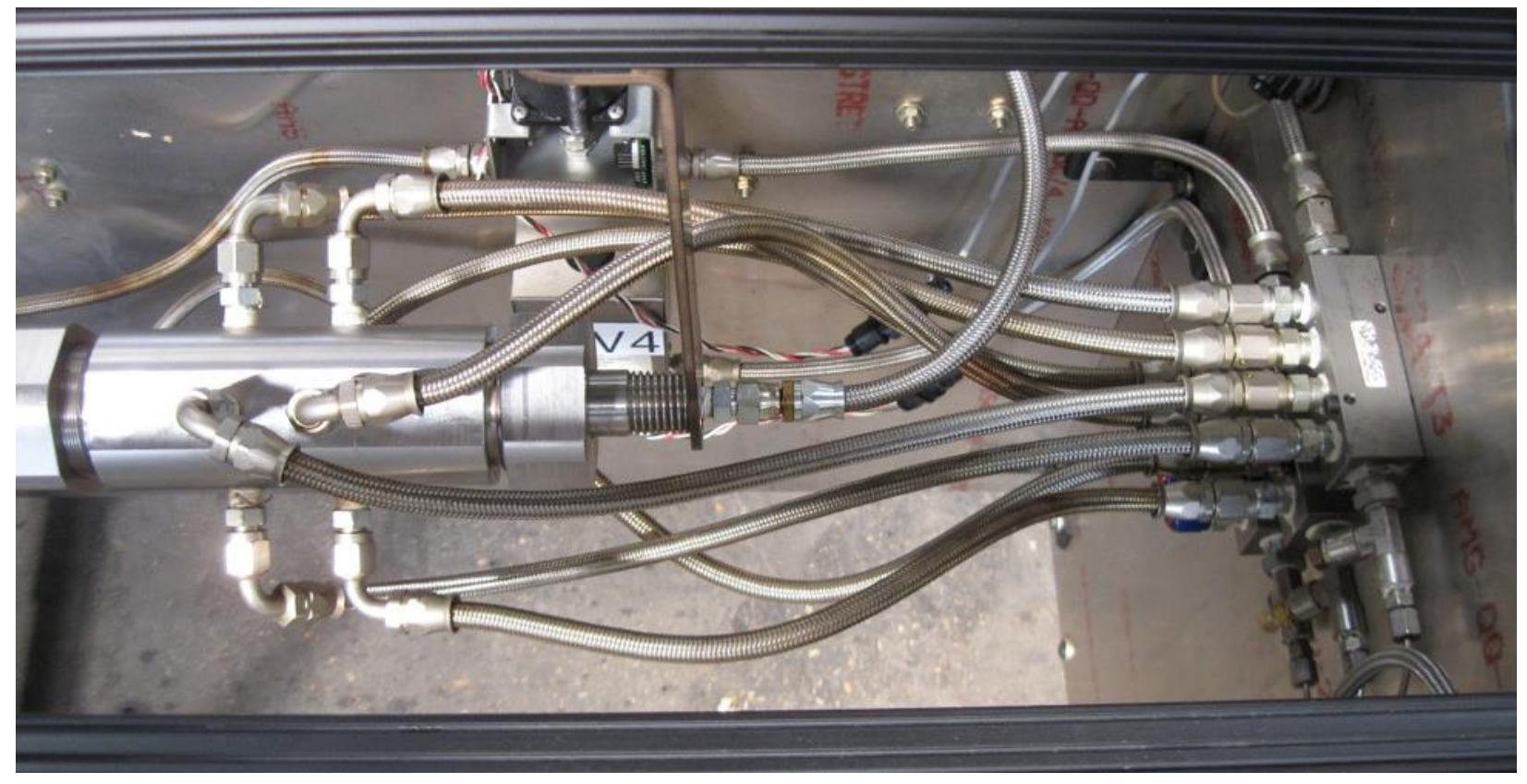

Figure 2, - an example of a composite technical solution (developed integrative technical system) designed to optimize the hydrodynamic parameters of the fluid flow in a pipeline, including stabilization of the basic and auxiliary hydrodynamic parameters of the fluid flow in a developed turbulent flow, installed on a dynamometer test bench at the following test phase for subsequent monitoring of correctness of the adopted fundamental design solutions, and harmonization of the results of implementation of these developed technical systems for compliance with the criteria - $a$ perfect end result.

It should be noted that the various organizational and economic levels of commercial structures where the concept generation takes place, the creation and implementation of such technical systems may differ significantly and depend on the size of the economic structure implementing the innovative concept.

At the same time, many examples have emerged of how the structure and system of a modern large enterprise negates innovative projects and focuses, as it seems at the time of decision-making, on the most effective innovative ideas, often neglecting equally effective solutions whose inventors and authors get (often undeservedly) severe psychological trauma.

An integrated systemic approach to the consistent, balanced, and accurate formation of such an innovative integrative technical solution or to a group of local unified innovative ideas connected by a 
single innovative integrative concept, which together constitute a systemic object of a complex integrative invention, is very important as well.

The systemic approach is a reflection and development of the dialectic principles of the "universal interconnection" and "development" and, in fact, is one of the principles of the dialectical method of cognition.

The systemic approach methodology from the point when the Algorithm for Inventive Problem Solving emerged implies the representation of any object in the form of various system variants and its comprehensive consideration.

Such an analysis requires clarifying the wording and definitions of the system and its components.

\section{The System}

The system, in the modern view, is a complex of elements, consistently organized in space and time, interconnected with each other and forming a certain integral unity.

The system is characterized by the composition of the elements, structure and performance of a specific function.

A significant addition to the concept of the system in modern conditions is the program and processor component of the system, taking into account vertical and horizontal components of integration of the elements of its structure as well.

\section{Elements}

Elements are relatively indivisible parts of the whole, objects that form a system together. An element is considered indivisible within the limits of preservation of a certain given quality of the system.

The new commonality of elements is the degree of their autonomy with regard to additional possibilities of autonomization with the help and with the participation of program and processor tools.

\section{The Structure}

The structure is a regular, stable relationship between the system elements, reflecting the form, the method of arrangement of the elements, and the nature of interaction of their features and properties. 
The structure makes the system a certain qualitatively defined whole, different from the sum of the qualities of its constituent elements (since it involves interaction of the elements with each other in different ways, only by certain features and properties, not as a whole).

More accurate classification of the structure requires formulating the definition of the limits of influence and autonomy related to the field of influence on these parameters by the processor and program components.

The capabilities of the processor and program components of the structure in combination with the traditional constituent elements to determine the levels of compliance of such a complex structure with the criteria of artificial intelligence today also create new limits for the criteria for assessing the general level of the structure and its compliance with the averaged technical requirements and conditions comparable to the definition of a perfect end result.

\section{The Function}

The function is an external manifestation of an object (element) properties in a given system of relations, taking into account the activity and influence of processor elements and, additionally, the functions of artificial intelligence. Such a complex function is a certain way of the object interaction with the environment, the object's "capability" of systemic interaction. Systems have many functions.

\section{Subsystems}

Subsystems (constituent systems) are parts of the system that represent some arbitrarily or naturally distinguished groups of elements, now often functionally connected by processor and program elements. Subsystems are allocated by functional features.

A single element can sometimes coincide with a certain subsystem or be a part of several different subsystems at once.

At the same time, the relationship between the elements within subsystems and within the system differs from the nature of the relationship between subsystems themselves. Elements and subsystems are united by the concept of a system component.

\section{The Supersystem}


The supersystem (metasystem) is a system of a higher order in relation to the given one, and where this system is included and functions as a subsystem.

Today, as a rule, the supersystem is defined and characterized by the level of the computer control and monitoring system, again, the relationships between the subsystem integration levels and flexibility, and the capability of self-control and systemic reorganization of the functional characteristics.

At present, the capabilities of remote control and contactless methods of measurement and monitoring of parameters in real time also characterize the classification and quality level of the supersystem.

\section{The Technical System}

The technical system (TS) is an artificially created material unity of interrelated elements naturally organized in space and time, with the aim of its functioning to satisfy a certain social need while maintaining a certain level of autonomy. TS elements can be both artificial and natural.

Any TS is part of two systems of relationships. On the one hand, it is an object of the material world that obeys the laws of nature (first of all, the laws of physics as the most common); on the other hand, TS acts as an element of public relations because technology and especially computer and processor technology are only means for social goals.

If TS is characterized by a spatial arrangement of elements, then TS is a device or substance. All technical systems, regardless of the nature of spatial arrangement of the elements, should now have inherent processor and program elements and real-time monitoring and control systems based on them.

If TS is characterized by organization of the elements in time, we are dealing with a method, although we should note the fact that today only one method that is not related functionally and by causal factors to a specific and effect relationship is not recognized as an invention.

The concept of TS allows to formulate the main feature of the technical solution (TSL).

TSL indicates a specific TS which functioning allows to achieve the goal set, that is, indicates the relationship of TS to a certain goal.

From the standpoint of systems engineering, TS can be represented as:

INPUT - PROCESSOR - OUTPUT. 
The processor converts the input to the output and at the same time is a component (constant) of the input.

INPUT and OUTPUT reflect the system interaction with the environment. From the physical point of view, TS has space, time, mass, energy, and information at the output and input.

From the socio-technical point of view, at the input we have the "needs" of TS - the costs of society for its creation, and at the output - the TS "capabilities", their main part is the functions of such system.

Acting in the form of the action of TS function in unity, the composition, and structure of the system's activities are determined, showing what TS can do: move in space, provide heating, and resist the wind.

The remaining "capabilities" characterize how the actions are taken: reliably, retaining the possibility of repair and regeneration, etc.

One can select the appropriate subsystem for each function in TS.

\section{Useful Functions}

Useful functions (UF) are functions that correspond to the system purpose, characterizing the most important constituent useful outputs.

In real TS, not all of the output is useful.

The usefulness of a particular part of the TS output can only be determined based on social positions. Those "capabilities" of TS that correspond to its purpose, i.e., social needs at the supersystem level, are useful.

Other capabilities may be useless or harmful, and that which actively interferes with the performance of useful "capabilities" is considered harmful, for example, by destroying TS elements, etc.

\section{New look at the new product reliability}

If we follow the above principles and definitions, then we should consider such a fundamental issue in new, especially innovative and combination developments as reliability and durability, through the 
prism of definitions and classifications at the first stage of the supersystem, then along the chain - the subsystem, then the technical system, then the structure and then the function.

Since reliability of computer technology and mechanical elements of any technical system quite differ, we should pay great attention to the calculations of reliability of such combined systems which include integrative inventions, computer simulation systems, and elements of artificial intelligence, and at the same time, the methodology of such calculations and analytical assessments should be thoroughly worked out by elements in certain and real production conditions comprising similar elements of the above chain of definitions and classifications at the first stage of the supersystem, then along the chain the subsystem, then the technical system, then the structure, and then the function.

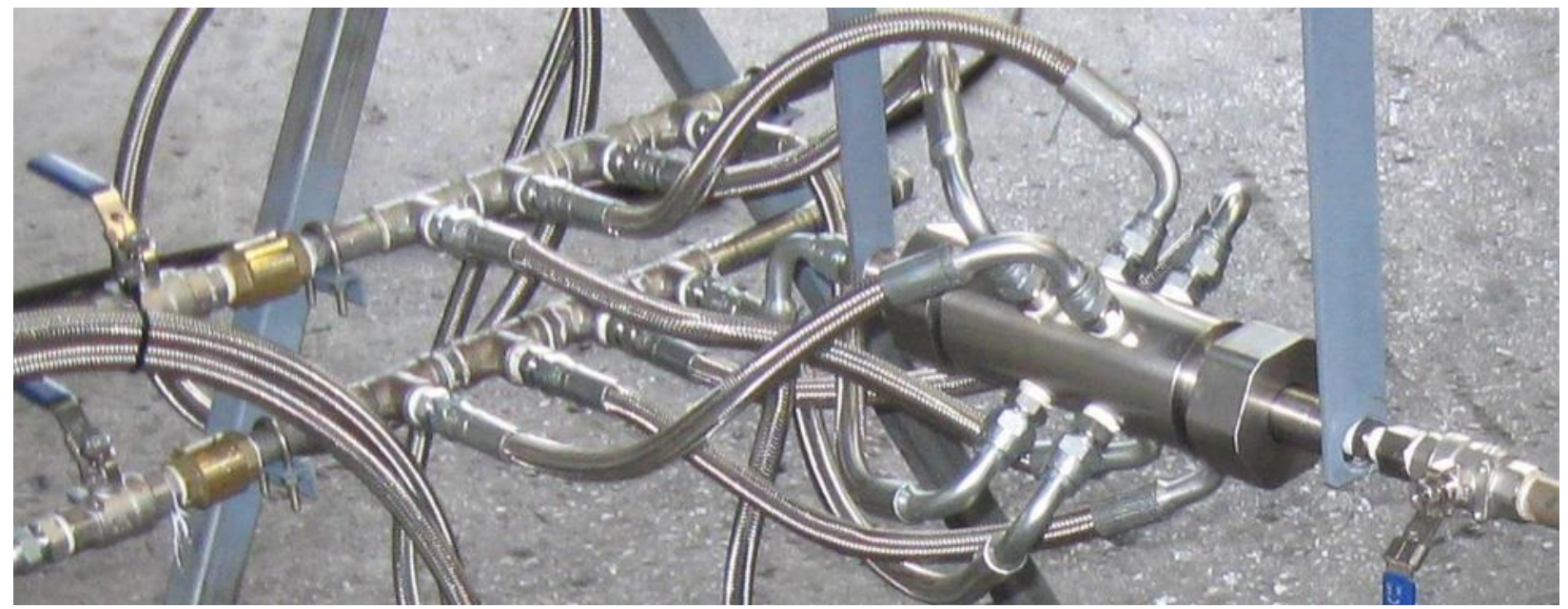

Figure 3, - an example of a composite technical solution (developed integrative technical system) designed to optimize the hydrodynamic parameters of the fluid flow in a pipeline, including stabilization of the basic and auxiliary hydrodynamic parameters of the fluid flow in a developed turbulent flow at the next stage of technological inspections, production tests for the subsequent optimization of constructive and technological solutions for the production of prototypes, consistent operational control of the correctness of the chosen basic design solutions, and technologies and equipment for the production.

As practice shows, in any project of any innovation and composition structural level, only a properly organized pilot industrial operation can provide the final answer in the development of any 
innovation project and in a comparative analysis and evaluation of its commercial and innovation potential.

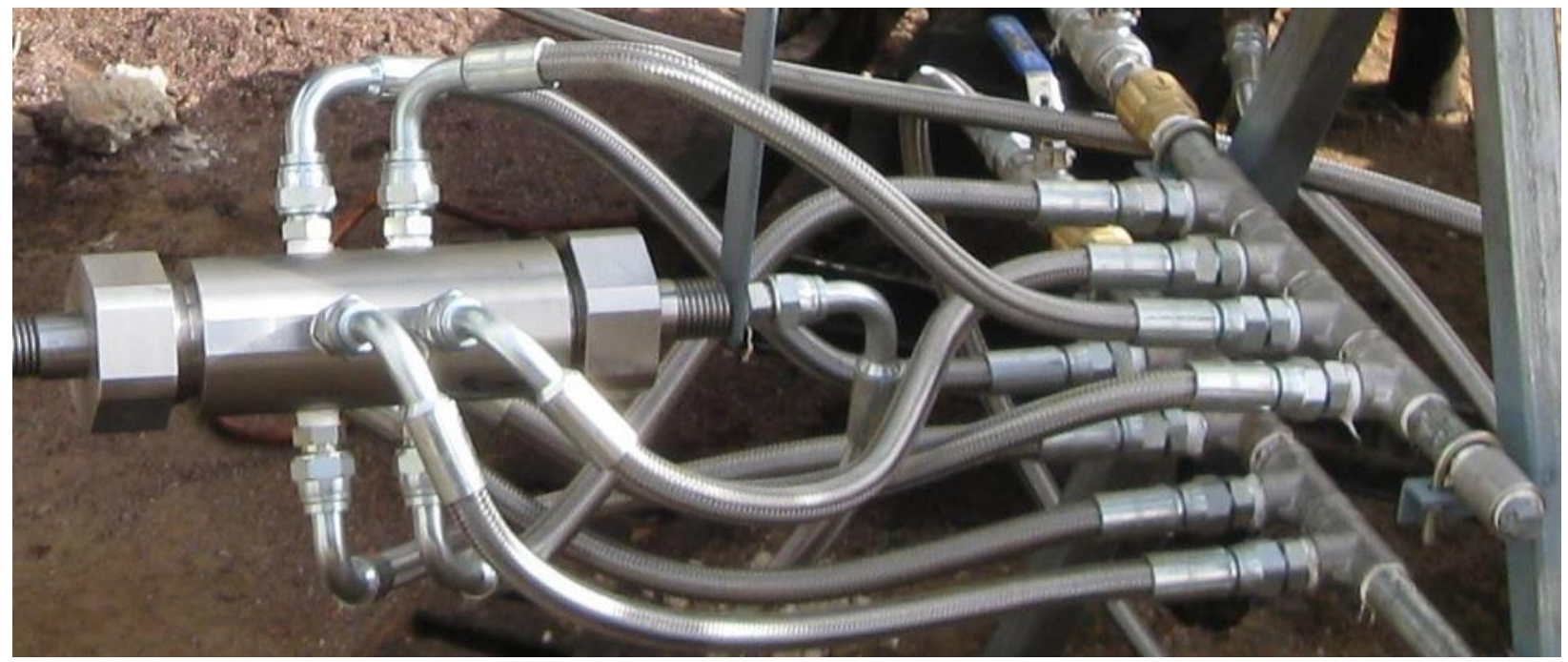

Figure 4, - an example of a composite technical solution (developed integrative technical system) designed to optimize the hydrodynamic parameters of the fluid flow in a pipeline, including stabilization of the basic and auxiliary hydrodynamic parameters of the fluid flow in a developed turbulent flow in real production tests and on test benches at the next complex production stage of testing for the final stage of subsequent monitoring and analytical evaluation of the correctness of the chosen basic design solutions and technological solutions.

Technical creativity and its highest manifestation - inventive activity aimed at creating original self-sufficient technical solutions - which became the basis of effective inventions demanded by society, requires tremendous work and creative impulse, time expenditures incommensurable with the time spent on only the technical or technological components of any project and more - an innovative project with signs of an integrative invention.

In the modern market conditions, when the final stage of the actual use of the invention can only be a product with a steady demand, the need for cooperation with specialists in the complex commercialization of innovations becomes a reality for practical inventors. 
As the practice of the last decade shows, the most commercially successful are technical solutions not focused on the already existing and well-understood consumer demand, but on forming a new, unusual, previously unknown demand.

Since the technical level of engineering and technology is constantly growing in such, as a rule, integrative and complex solutions, following the laws, postulates and principles of the Theory of Inventive Problem Solving and the Algorithm for Inventive Problem Solving requires a constant comparison of existing provisions with newly emerged circumstances.

And even after realizing the need for systemic modification and optimization of the ideological, estimate and technical-technological base of such multi-stage cooperation between the generators of ideas - technical and innovative interpreters of global and even partially abstract ideas into really solved and realizable technical solutions, after realizing the need for deep cooperation with specialists in commercialization of new technological ideas and solutions, there remain specific, but in principle absolutely strategic issues that can be solved only by the inventor, who is the best to understand and know the features of his or her invention.

It seems to the author of this paper that just to help inventors in the process of identifying and deepening their understanding of the capabilities and peculiarities of their inventions, adaptation of the Theory of Inventive Problem Solving and the Algorithm for Inventive Problem Solving to modern conditions can provide all the necessary analytical tools.

The emergence of new markets, domestic and external, and the development of an economic organization from a craft workshop and factory to concerns such as U.S. Steel, illustrates the same process of economic mutation - if the biological term can be used here - which is constantly revolutionizing... the economic structure from the inside, destroying the old structure and creating a new one.

This process of "creative destruction" is the very essence of modern production.

Every enterprise that introduces innovations has to exist within its framework.

The behavior of an enterprise should be assessed only against the background of the overall process, in the context of the situation it generated. It is necessary to clarify its role in the constant stream of "creative destruction". It is impossible to understand it outside this stream. 
In the future series of papers offered to readers, the author set a goal of relating future work on the commercialization of any invention with the initial stages of working on its creation, especially at the stages of generating ideas.

The inventor, who begins the process of forming basic ideas and principles for creating his or her future invention, even without knowing the principles of the Theory of Inventive Problem Solving and the Algorithm for Inventive Problem Solving, whether wanting it or not, uses them, even intuitively.

At the same time, the Theory of Inventive Problem Solving and the Algorithm for Inventive Problem Solving in their historical and classical edition do not help the inventor to decide on the estimated commercial value of his or her future invention.

In contrast to repeatedly practically proven and unique methods and principles, laws of technical systems development, which are basic for the Theory of Inventive Problem Solving and the Algorithm for Inventive Problem Solving, the methods of commercialization and analysis of the potential capabilities of inventions as commercial products, do not yet have a systemic theoretical basis.

Recently, many publications have appeared to provide recommendations on commercialization, and a decision has been made based on them to use one of them, to, firstly, provide the inventors with real-time information which they can, if they wish, adapt with the technical characteristics and advantages of their invention, and especially important, secondly, to show one of the possible versions and principles of practical actions showing how to change and adjust the technical characteristics and parameters of the future innovation development depending on market requirements.

Taking into account the conditions and all sorts of restrictions formed by the features of the innovation process in the mode if globalization of the world economy, it can be assumed that, namely, the systemic unification of the elements of innovative products at the component level can help to gradually form a library of components, assemblies, and basic parts, which the inventors can use to form the technological embodiment of their ideas.

It seems to the author of this publication that taking into account possible market requirements at the stage of generating an innovative idea will enable forming such a technical characteristic of a new product that will contribute to a more confident and cost-effective introduction of the innovation, but if the assessment of the commercial significance of the generated idea is low, giving up this idea and paying 
attention to something else, or by modulating and unifying the idea, turning the idea in a cost-effective direction.

In his papers on the Theory of Inventive Problem Solving and the Algorithm for Inventive Problem Solving, the author had to repeatedly note the indisputable fact that most of the inventions being created now are integrative because any modern effective technical solution have both digital control systems, composite materials, nano-coatings, and various integrative combinations of the program, system, method, and apparatus type.

For such complex, combined and integrated systems, the principles of a system analysis of their commercial value have yet to be formulated, and publication of information about the methods, techniques, and working schemes of commercialization will help inventors working in the field of innovative projects today.

Impossibility of successful commercialization without forming the principles of the compositional interactions and the compositional structure of a new technical solution.

The primary insight into a new product in the market as a composition technical solution within the Technical System and within the framework of interrelations comprising a supersystem with processor and computer elements inherent in modern innovative products cannot be imagined without an appropriate presentation format via the Internet and relevant websites using all innovative information presentation, modeling, and simulation capabilities.

Thus, the success of the first stages of commercialization largely depends on the success of such a presentation. 


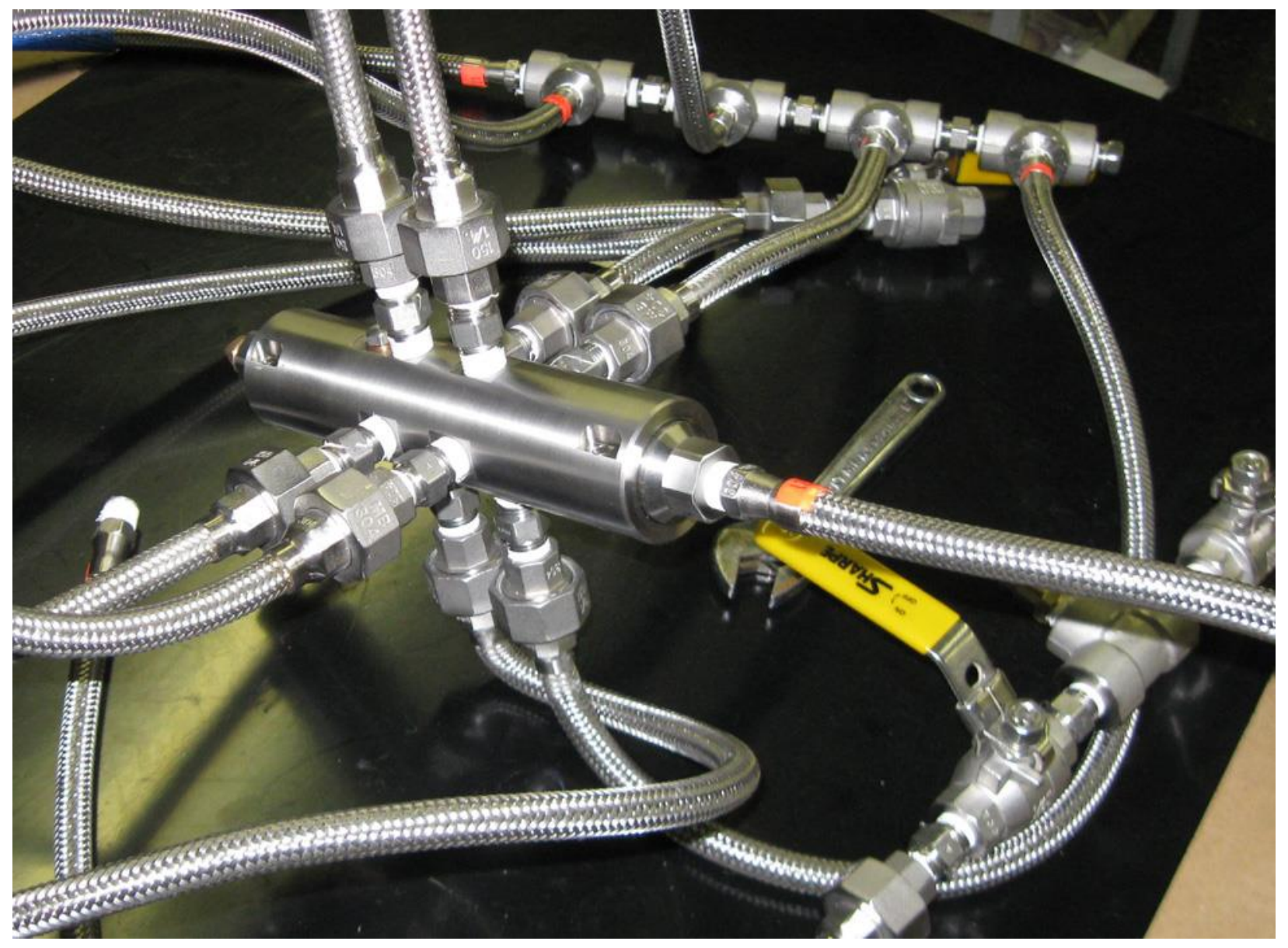

Figure 5, - an example of a composite technical solution (developed integrative technical system) designed to optimize the hydrodynamic parameters of the fluid flow in a pipeline, including stabilization of the basic and auxiliary hydrodynamic parameters of the fluid flow in a developed turbulent flow at the next stage of testing after the final design stage and after applying numerically controlled production methods, for subsequent control of the correctness of the chosen basic design solutions, methods, and equipment for the production.

The proposed techniques and methods for formation of a compositional style in creation of new innovative solutions

As can be seen from the above, the systemic bases for defining the criteria of compositional style as a direction for creating new innovative solutions and technical systems are in the process of defining and forming, and there is every reason to believe that the Theory of Inventive Problem Solving and the Algorithm for Inventive Problem Solving will play the key role in this dynamic process. 


\section{References}

1. US patent application No20180308005, 10.25.2018. ARTIFICIAL INTELLIGENCE PROFILING / Banipal; Indervir Singh; et al.

2. US patent application No20180144067, 05.24.2018. CLOSED-LOOP UNIFIED METADATA ARCHITECTURE WITH UNIVERSAL METADATA REPOSITORY / Chatelain; Jean-Luc; et al.

3. US patent application No20180129785, 05.10.2018. SYSTEMS AND METHODS FOR TECHNICAL SUPPORT OF CONTINUOUS ANALYTE MONITORING AND SENSOR SYSTEMS / Pal; Andrew Attila; et al.

4. US patent application No20180232587, 08.16.2018. VEHICLE MONITORING METHOD AND APPARATUS, PROCESSOR, AND IMAGE ACQUISITION DEVICE / WANG; Qun; et al.

5. US patent application No20180030398, 02.01.2018. SYSTEM, APPARATUS AND METHOD FOR THE PRODUCTION OF CELLS AND/OR CELL PRODUCTS / Castillo; Jose.

6. US patent application No20180013633, 01.11.2018. INDUSTRIAL SETUP COMPOSITION / Daijavad; Shahrokh; et al.

7. US patent application No20170344673, 11.30.2017. METHOD AND APPARATUS FOR PROVIDING A SAFE OPERATION OF A TECHNICAL SYSTEM / Hofig; Kai; et al. 